Bundesgesundheitsbl - Gesundheitsforsch Gesundheitsschutz 2007 · 50:718-727 DOI 10.1007/s00103-007-0233-7

c) Springer Medizin Verlag 2007

H. Kahl ${ }^{1}$ - R. Dortschy ${ }^{1}$ - G. Ellsäßer ${ }^{2}$

${ }^{1}$ Robert Koch-Institut, Berlin, BRD . ${ }^{2}$ Landesgesundheitsamt Brandenburg,

Zossen, BRD

\title{
Verletzungen bei Kindern und Jugendlichen (1-17 Jahre) und Umsetzung von persönlichen Schutzmaßnahmen
}

\section{Ergebnisse des bundesweiten Kinder- und Jugendgesundheitssurveys (KiGGS)}

\section{Hintergrund}

Unfälle sind die häufigste Todesursache bei Kindern ( $>1$ Jahr) und Jugendlichen in Deutschland und Europa und sind die Hauptursache für die Krankheitslast gemessen an DALYs (years of life lost to disability or premature death) $[1,2]$. Nach der Studie von UNICEF liegt der Anteil der tödlichen Verletzungen an allen Todesfällen im Kindesalter in Europa bei ca. $40 \%$. Europaweit könnten allein jährlich 1600 tödliche Unfälle im Kindesalter vermieden werden, wenn man die Maßnahmen, die zum niedrigen Stand der tödlichen Unfälle in Schweden beigetragen haben, zum Maßstab nehmen würde [3]. Unfälle sind somit ein vorrangiges bevölkerungsmedizinisches Problem und haben weitreichende individuelle und soziale, aber auch ökonomische Folgen [4, 5].

Eine im Jahr 2006 publizierte Studie zur Epidemiologie von Unfällen bei Kindern unter 15 Jahren in Deutschland, die die bundesweit verfügbaren Datenquellen für den Zeitraum von 1990-2004 epidemiologisch aufbereitete, kam zu folgenden Schlussfolgerungen: In Deutschland ging die Unfallmortalität von Kindern unter 15 Jahren im Heim- und Freizeitbereich als auch Verkehrsbereich seit 1990 stark zurück (range 68-72\%). Säuglinge und kleine Kinder sowie Jungen ab dem zweiten Lebensjahr hatten das höchste Risiko, an einem Unfall zu sterben bzw. sich schwer zu verletzen. Nur bei den unter 5Jährigen nahm die Rate der im Krankenhaus behandelten Fälle stark zu. Am häufigsten verletzten sich die unter 5 -Jährigen zu Hause, während bei den 5- bis 14-Jährigen die 3 häufigsten Unfallorte die Schule, der Heim- und Freizeitbereich und der Straßenverkehr waren. Insgesamt wurde die Anzahl der verletzten Kinder unter 15 Jahren für das Jahr 2004 auf 1,6 Millionen geschätzt [6].

Die Studienergebnisse über ein 4-jähriges Monitoring (1998-2002) von verletzten Kindern, die in den Notfallambulanzen bzw. Krankenhäusern der Stadt Delmenhorst behandelt werden mussten, zeigten des Weiteren, dass sich Kinderunfälle alters- und umgebungsspezifisch ereigneten. Stürze waren mit über $50 \%$ Anteil der häufigste Unfallmechanismus in allen Altersgruppen und standen bei der Hälfte aller Unfälle mit Produkten im Zusammenhang: im Säuglingsalter am häufigsten Stürze vom Wickeltisch, gefolgt von Stürzen aus dem Kinderbett, im Kleinkindalter dagegen vorrangig Stürze von Spielplatzgeräten und im Schulalter
Stürze vom Fahrrad, gefolgt von Stürzen von Spielplatzgeräten [7].

$\mathrm{Zu}$ Unfällen und sozioethnischen Risiken gibt es in Deutschland nur wenige bevölkerungsbezogene Studien. Einen wesentlichen Beitrag leisten hier die kinderärztlichen Einschulungsuntersuchungen des Öffentlichen Gesundheitsdienstes, wenn sie Informationen zum Unfallort und zu den Verletzungsdiagnosen kindbezogen mit dem Sozialstatus der Eltern verknüpfen (Brandenburg, Schleswig-Holstein) $[8,9]$. So zeigten die Brandenburger Analysen, dass seit Jahren eine erhöhte Unfallgefährdung bei Kindern in Familien mit niedrigem Sozialstatus für Straßenverkehrsunfälle und Verbrühungen bestand, jedoch nicht für andere Unfallorte oder Verletzungsfolgen $[10,11]$. Die Daten des Delmenhorster Unfallmonitorings konnten den starken Einfluss des Migrationsstatus nachweisen. Verletzungen insgesamt und besonders ausgeprägt Verbrühungen kamen in Migrantenfamilien um ein Vielfaches häufiger vor als in deutschen Familien $[12,13]$. Analoge Ergebnisse wurden in internationalen Studien beschrieben und auf die Zugehörigkeit zu ethnischen Minderheiten sowie auf einen niedrigeren sozioökonomischen Status zurückgeführt 
$[14,15,16,17,18]$. UNICEF wies darüber hinaus für thermische Verletzungen auf folgende Einflussfaktoren hin: Armut, Familienstand, geringer Bildungsgrad, Arbeitslosigkeit, niedriges Alter der Mutter, ärmliche Wohnverhältnisse, kinderreiche Familien sowie elterlicher Drogen- und/ oder Alkoholkonsum [3].

\section{Datenlage zu Unfällen und persönlichen Schutzmaßnahmen bei Kindern in Deutschland}

In Deutschland sind nur über Unfälle mit Todesfolge auf der Grundlage der Todesursachenstatistik Aussagen zu Hochrisikogruppen und Unfallschwerpunkten möglich. Denn hier werden für jeden Todesfall demografische Daten (Alter, Geschlecht, Nationalität, Wohnort) und nach ICD-10 die „äußeren Ursachen“ (Kapitel XX ICD-10), der Unfallort und die Unfalltätigkeit kodiert. Soziale Merkmale werden im Gegensatz zu anderen europäischen Ländern wie Dänemark oder England nicht erfasst [6]. Lückenhaft ist jedoch die Datenlage zu Verletzungen im Kindes- und Jugendalter. Im Gegensatz zu anderen europäischen Ländern wie den Niederlanden, Dänemark, Wales/UK, Italien gibt es für Deutschland keine systematische bevölkerungsbezogene Erfassung (Monitoring) von Verletzten und daher keine repräsentativen Daten zu Kinderunfällen [6, 19]. Zu Verletzungen, die eine Krankenhausbehandlung notwendig machen, sind bundesweit Diagnosen nach ICD-1o über die amtliche Krankenhausdiagnosestatistik verfügbar, aber weitere für die Prävention bedeutsame Merkmale wie Unfallort und Unfallmechanismus werden nicht erfasst. Aktuelle Teilinformationen liegen bundesweit für einzelne Unfallkategorien wie den Straßenverkehr und die Bildungsund Betreuungseinrichtungen vor [20]. Für den Heim- und Freizeitbereich wurden die seit 1988 4-jährig durchgeführten repräsentativen Surveys im Jahr 2000 eingestellt [6]. Auf Länderebene sind die kinderärztlichen Einschulungsuntersuchungen in Brandenburg und SchleswigHolstein die einzigen bevölkerungsbezogenen Erfassungssysteme in Deutschland, die die Lebenszeitprävalenz von Unfällen bei Einschulungskindern unter Einbezie- hung sozialer Merkmale kontinuierlich erfassen und bewerten $[8,9]$.

Ähnlich dünn ist auch die Datenlage zu den persönlichen Schutzmaßnahmen von Kindern und Jugendlichen, z. B. über das Tragen eines Helmes beim Fahrradfahren oder Skaten bzw. das Tragen von Schutzkleidung wie Arm- und Knieschoner beim Skaten. Die letzten publizierten Untersuchungsergebnisse der Bundesanstalt für Straßenwesen gehen auf das Jahr 2005 zurück und wiesen eine Helmtragequote von $53 \%$ bei den 10-jährigen Rad fahrenden Kindern nach. Daten zum Skaten wurden bisher nicht erhoben [21, 22].

\section{Unfalldefinition}

Die Definition von Verletzungen durch die Weltgesundheitsorganisation (WHO) [23] unterscheidet zwischen den unbeabsichtigten (unintentional) und beabsichtigten (intentional) Verletzungen. Die unbeabsichtigten Verletzungen sind die sog. „Unfälle“ im engeren Sinne. Beabsichtigte Verletzungen sind dagegen solche, die durch Gewalt von außen, durch interpersonale Gewalt oder durch Gewalt gegen sich selbst (Selbstverstümmelung, Suizidversuch) hervorgerufen werden. Publikationen, die diese Definition berücksichtigen und die verfügbaren Daten für das Kindes- und Jugendalter entsprechend epidemiologisch bewerten, gibt es in Deutschland nur wenige [6, 7]. Säuglinge hatten das höchste Risiko, durch Gewalt zu sterben, im Vergleich zu Kleinkindern (ein bis 4 Jahre) und Schülern (5-14 Jahre). Der Anteil der tödlichen Verletzungen durch Gewalt an den Verletzungen insgesamt war bei den Säuglingen mit über $50 \%$ am höchsten [6]. Bei der Auswertung der Daten des Kinderund Jugendgesundheitssurveys (KiGGS) wurde daher die WHO-Definition von Verletzungen zugrunde gelegt. Eine Unterscheidung der Verletzungen insgesamt durch Unfälle und Gewalt war möglich, da Eltern und Kinder ab 11 Jahren auch nach Verletzungen durch tätliche Auseinandersetzungen befragt wurden.

Aufgrund der lückenhaften Datenlage $\mathrm{zu}$ Verletzungen bei Kindern und Jugendlichen ist ein vorrangiges Ziel des KiGGS, repräsentative Daten zu Verletzungen und zu Verletzungsmechanismen durch Un- fälle und tätliche Auseinandersetzungen bei Kindern und Jugendlichen zu erhalten. Darüber hinaus werden altersspezifische Informationen zur Umsetzung der persönlichen Schutzmaßnahmen wie Helmtragequoten benötigt, um die Wirkung bisheriger Präventionsstrategien zu bewerten.

\section{Methoden}

In den ersten 6 Beiträgen in diesem Heft werden Konzept, Design und Durchführung des Kinder- und Jugendgesundheitssurveys (KiGGS) ausführlich beschrieben [24, 25, 26, 27, 28, 29, 30]. Die KiGGS-Studie wurde von Mai 2003 bis Mai 2006 durch das Robert Koch-Institut (RKI) durchgeführt. Ziel dieses bundesweiten Befragungs- und Untersuchungssurveys war es, erstmals umfassende und bundesweit repräsentative Daten zum Gesundheitszustand von Kindern und Jugendlichen im Alter von o-17 Jahren zu erheben. An der Studie haben insgesamt 17.641 Kinder und Jugendliche (8656 Mädchen und 8985 Jungen) aus 167 für die Bundesrepublik repräsentativen Städten und Gemeinden teilgenommen. Die Teilnahmequote betrug insgesamt 66,6\%. Um repräsentative Aussagen treffen zu können, wurden die Analysen mit einem Gewichtungsfaktor durchgeführt, der Abweichungen der Netto-Stichprobe von der Bevölkerungsstruktur (Stand: 31.12.2004) hinsichtlich Alter (in Jahren), Geschlecht, Region (Ost/West/Berlin) und Staatsangehörigkeit korrigiert. Im Survey wurden bei den unter Einjährigen die Kinder nicht erfasst, die nach dem Zeitpunkt der Stichprobenziehung aus den Einwohnermeldeamtsdaten geboren wurden. Hierbei handelt es sich um Säuglinge, die jünger als 3 Monate sind. Zur Gewährleistung repräsentativer Aussagen auch für die Altersgruppe der unter Einjährigen wurde dies in der Gewichtung entsprechend berücksichtigt.

Ausgangspunkt für die Erfassung der Unfalldaten war folgende zentrale Frage im Elternfragebogen: „Hat sich Ihr Kind in den letzten 12 Monaten durch einen Unfall verletzt oder vergiftet und musste deshalb ärztlich behandelt werden?" (Antwortkategorie „ja“, „nein“.) Dabei sollten die Eltern bei mehreren Unfällen des Kin- 
des nur den zeitlich letzten Unfall berücksichtigen. Wurde eine ärztlich behandelte Verletzung bejaht, sollten 4 weitere Fragenbereiche zum Unfallort, zum Unfallmechanismus, zu den Verletzungsfolgen und zur ambulanten bzw. stationären Behandlung beantwortet werden.

Die Responsequote der Eltern der untersuchten Kinder und Jugendlichen von 1 bis unter 18 Jahren war für die einzelnen Fragenbereiche wie folgt: für die Ausgangsfrage $97,7 \%$ (16.327 von 16.706), darunter Migranteneltern 90,4\% (2242 von 2479); für den Unfallort bezogen auf 2541 verletzte Kinder (1-17 Jahre) 95,7\% (2433 von 2541); zu Unfallmechanismen 94,9\% (2413 von 2541) und zu Verletzungsfolgen 90,1\% (2290 von 2541). Eine ebenso hohe Responsequote von $96 \%$ bzw. 92,2\% erreichten die Elternangaben bei den Fragen zu persönlichen Schutzmaßnahmen der Kinder im Alter von 3-17 Jahren, d. h. Helmtragen beim Fahrradfahren und Inlineskaten bzw. der Einsatz von Knie- und Armschonern. Auch das Antwortverhalten der befragten 11- bis 18-jährigen Kinder und Jugendlichen (6813) zum Einsatz persönlicher Schutzmaßnahmen lag für die 3 identisch zum Elternfragebogen gestellten Fragen bei 94\%. Die Datenanalyse wurde aus oben erwähnten methodischen Gründen altersgruppenbezogen erst ab dem ersten Lebensjahr durchgeführt. Die Analyse umfasst auf dieser Grundlage die Darstellung von Häufigkeiten nach Verletzungen durch Unfälle und Gewalt, Unfallort, Verletzungsmechanismus, Verletzungsfolgen, ambulanter bzw. stationärer Behandlung und Helmtragequoten beim Fahrradfahren bzw. Inlineskaten. Darüber hinaus erfolgte eine bivariate Analyse der Zielvariablen nach Sozialund Migrationsstatus. Um die möglichen Merkmalskorrelationen innerhalb einer Gemeinde zu berücksichtigen, wurden die Konfidenzintervalle und die p-Werte mit dem SPSS-14-Verfahren für komplexe Stichproben bzw. mit den Survey-Prozeduren von SAS 9.1 bestimmt.

\section{Ergebnisse}

\section{Verletzungen}

Insgesamt hatten von 16.327 Kindern und Jugendlichen (1-17 Jahre) $15,9 \%$ in

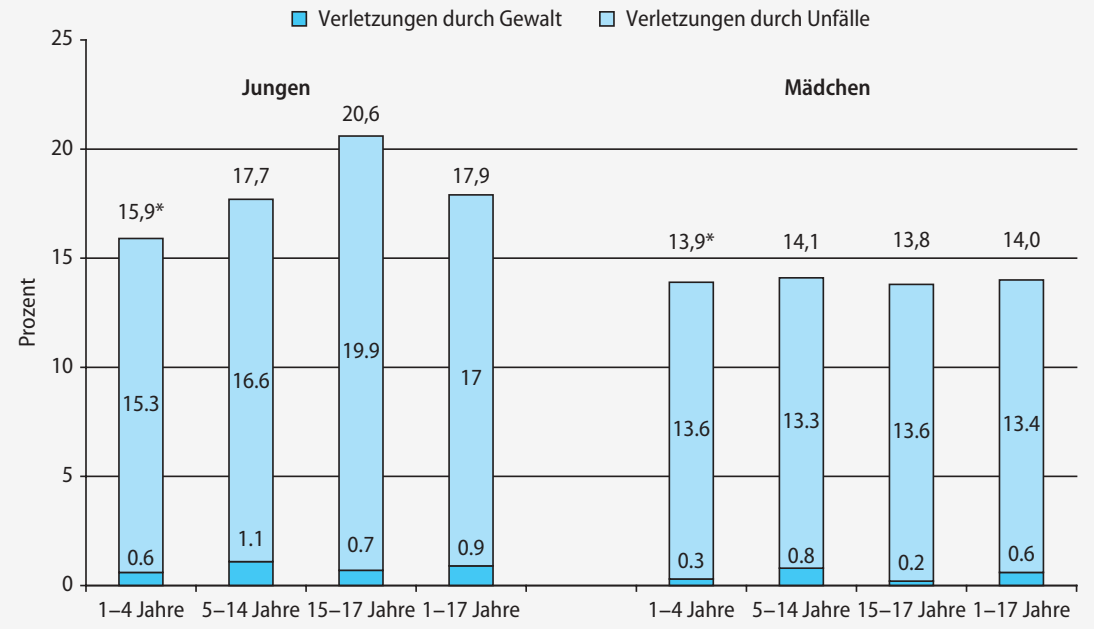

Abb. $1 \Delta$ Jahresprävalenzraten von Verletzung durch Unfälle bzw. durch Gewalt bei Kindern und Jugendlichen (1-17 Jahre) nach Geschlecht und Altersgruppen $(n=16.327)$.

* Eine tätliche Auseinandersetzung bei Kindern unter 5 Jahren wird unter „Unfall“ kategorisiert, da eine Absicht nicht unterstellt werden kann. Dieses Vorgehen entspricht den Definitionen im Kodierungsmanual der Injury Data Base (IDB) [35].

den letzten 12 Monaten mindestens eine Verletzung erlitten, darunter $15,2 \%$ durch Unfälle und o,8\% durch Gewalt. Verletzungen betrafen Jungen im Alter von ein bis unter 18 Jahren signifikant häufiger als Mädchen (17,9\% vs.14,0\%). Dieser geschlechtsspezifische Unterschied zeigte sich bei den ein- bis unter 18-Jährigen ebenso bei den Verletzungen durch Unfälle $(17,0 \%$ vs. $13,4 \%)$ wie bei den Verletzungen durch Gewalt (o,9\% vs. o,6\%) (• Abb. 1).

\section{Verletzungen durch Unfälle}

15,2 \% der Ein- bis unter 18-Jährigen hatten einen Unfall erlitten, darunter die 15- bis unter 18-jährigen Jugendlichen mit der höchsten Rate von 16,8\%. Während bei den Jungen ein kontinuierlicher Anstieg der Unfallraten vom Kleinkind- bis zum Jugendalter (von $15,3 \%$ auf $19,9 \%$ ) zu verzeichnen war, lagen die Unfallraten bei den Mädchen in allen Altersgruppen bei ca. $13 \%$. Der Geschlechtsunterschied war erst ab der Altersgruppe der 5- bis unter 15-Jährigen signifikant $(\mathrm{p}<0,001)$. Eine weitere statistische Analyse der Unfallhäufigkeit in den definierten Altersgruppen nach Region (Ost vs. West) und Sozialstatus (niedrig, mittel, hoch) ergab nur für Kleinkinder (1-4 Jahre) bezogen auf die Region Ost eine signifikante niedrigere Verletzungshäufigkeit.
13,3\% der verletzten Kinder und Jugendlichen (1-17 Jahre) mussten im Krankenhaus behandelt werden. Bei einem Vergleich der Altersgruppen zeigte sich, dass Kleinkinder die höchste stationäre Behandlungsrate von allen Altersgruppen aufwiesen (17,1\%). Die durchschnittliche Verweildauer lag bei knapp der Hälfte der Ein- bis unter 18-Jährigen bei ein bis $2 \mathrm{Ta}$ gen, ein Viertel musste 3-5 Tage und ein weiteres Viertel mehr als 5 Tage behandelt werden.

\section{Verletzungen durch Gewalt}

Verletzungen durch Gewalt bei tätlichen Auseinandersetzungen betrafen 0,9\% der ein- bis unter 18-jährigen Jungen und o,6\% bei den Mädchen (• Abb. 1). Bei einem Vergleich der Altersgruppen zeigte sich, dass männliche Schüler (5-14 Jahre) bei Verletzungen durch Gewalt mit einem Anteil von 6,3\% an den Verletzungen insgesamt den höchsten Wert erreichten. Keine signifikanten Unterschiede konnten für die Merkmale Geschlecht, Region, Sozial- und Migrantenstatus gefunden werden.

\section{Unfallorte}

Der häufigste Unfallort bei Kleinkindern war mit 60,0\% das Zuhause, einschließlich der nahen Wohnumgebung (Garten, 


\section{Zusammenfassung $\cdot$ Abstract}

Bundesgesundheitsbl - Gesundheitsforsch - Gesundheitsschutz 2007 · 50:718-727

DOI 10.1007/s00103-007-0233-7

(C) Springer Medizin Verlag 2007

H. Kahl· R. Dortschy · G. Ellsäßer

\section{Verletzungen bei Kindern und Jugendlichen (1-17 Jahre) und Umsetzung von persönlichen Schutzmaßnahmen. Ergebnisse des bundesweiten Kinder- und Jugendgesundheitssurveys (KiGGS)}

\section{Zusammenfassung}

Die Befragung der Eltern zu Unfällen ihrer Kinder und Unfall vorbeugenden Maßnahmen im Kinder- und Jugendgesundheitssurvey (KiGGS) verfolgt das Ziel, repräsentative alters- und geschlechtsspezifische Daten zu Verletzungen im Kindes- und Jugendalter zu erhalten, um Risikogruppen und Risikofaktoren für die Unfallprävention zu identifizieren. Befragt wurden 16.706 Eltern zu Verletzungen ihrer Kinder (1-17 Jahre) in den letzten 12 Monaten, die ärztlich behandelt werden mussten, und zu Merkmalen des Unfallgeschehens wie Unfallort, Unfallmechanismus, Verletzungsfolgen und ambulante bzw. stationäre Behandlung. Darüber hinaus wurden sowohl Eltern als auch Kinder und Jugendliche zwischen 11 und 17 Jahren $(n=6813)$ zu Schutzmaßnahmen befragt. 15,9\% der Kinder und Jugendlichen (1-17 Jahre) hatten nach Elternangaben mindestens eine Verletzung, davon 15,2\% durch einen Unfall und 0,8\% durch Gewalt bei tätlicher Auseinandersetzung. In der Altersgruppe der Ein- bis unter 18-Jährigen verunglückten Jungen signifikant ( $p<0,001$ ) häufiger als Mädchen (17,9\% vs. $14,0 \%$ ). Insgesamt mussten $13,3 \%$ von 2410 verletzten Kindern und Jugendlichen im Krankenhaus behandelt werden. Bei den Unfallorten dominierten die häuslichen Unfälle mit $60 \%$ bei den Kleinkindern (1-4 Jahre), während Sport- und Freizeitunfälle bei den 5- bis unter 15-Jährigen und 15- bis unter 18-Jährigen im Vor- dergrund standen (32,1\% bzw. 38,9\%). Der Anteil der Unfälle in den Betreuungsund Bildungseinrichtungen verdreifachte sich vom Kleinkindalter zum Schulalter (514 Jahre) von 10,9\% auf 28,7\% und ebenso der Anteil der Straßenverkehrsunfälle von 5,6\% auf 16,7\%. Die 3 häufigsten Verletzungsmechanismen bei Kindern und Jugendlichen (1-17 Jahre) waren Stürze in der Ebene (35,2\%), aus der Höhe (25,2\%) und Zusammenstöße (20,6\%). Stürze aus der Höhe hatten ihren Häufigkeitsgipfel im Kleinkindalter (35,8\%). Prellungen, Verrenkungen und Zerrungen erreichten im Jugendalter (15-17 Jahre) mit 50,9\% einen Höchstwert; ebenso nahm der Anteil der Knochenbrüche von 10,7\% bei Kleinkindern auf $21,8 \%$ bei den 15 - bis unter 18-jährigen Jugendlichen stark zu. Während ein Zusammenhang zwischen Unfällen und Sozialstatus bei den Unfällen insgesamt und einzelnen Verletzungsfolgen nicht festgestellt werden konnte, zeigten sich jedoch bei den Verkehrsunfällen für ein- bis unter 18-jährige Mädchen $(p=0,047)$ und Jungen $(p=0.019)$ signifikant höhere Raten bei niedrigem Sozialstatus der Eltern verglichen zum hohen Sozialstatus. Bei der Umsetzung von Schutzmaßnahmen lagen die Raten bei den 15- bis unter 18-jährigen Jugendlichen am niedrigsten. Während nach Elternangaben die 3- bis unter 5- jährigen Jungen und Mädchen hohe Helmtragequoten beim Fahrradfahren und Inlineskaten von ca. $90 \%$ erreichten, waren diese bei den 5- bis unter 15-Jährigen Jungen und Mädchen mit über $60 \%$ deutlich geringer und bei den 15- bis unter 18-Jährigen mit nur noch knapp $15 \%$ am niedrigsten. Ebenfalls waren auch die Tragequoten für Protektoren bei den 15- bis unter 18-jährigen Jugendlichen am niedrigsten (Jungen $41,8 \%$ vs. $52,2 \%$ bei Mädchen). Die Selbstangaben der 11- bis unter 18-Jährigen lagen deutlich unter den Elternangaben. Der Sozial- und Migrationsstatus zeigte in allen Altersgruppen einen signifikanten Zusammenhang mit niedrigen Tragequoten bezogen auf Helme und Protektoren. Die altersgruppenbezogene Datenanalyse sollte Ausgangspunkt für zielgruppenbezogene Präventionsmaßnahmen sein und insbesondere den Sozial- und Migrationsstatus berücksichtigen. Dabei sind Präventionsaktivitäten im Verkehrsbereich insbesondere auf Familien mit niedrigem Sozialstatus auszurichten. Jugendliche sollten bei der Aufklärung über den Nutzen von Schutzmaßnahmen beim Fahrradfahren und Skaten verstärkt und adäquat angesprochen werden.

\section{Schlüsselwörter}

Gesundheitssurvey · Kinder · Jugendliche . Verletzungen · Stürze · Sozialstatus . Migrationsstatus · Helmtragequoten Radfahren/Skaten · Protektoren 
Bundesgesundheitsbl - Gesundheitsforsch - Gesundheitsschutz 2007 · 50:718-727

DOI 10.1007/s00103-007-0233-7

๑) Springer Medizin Verlag 2007

\section{H. Kahl·R. Dortschy · G. Ellsäßer}

\section{Injuries among children and adolescents (1-17 years) and implementation of safety measures. Results of the nationwide German Health Interview and Examination Survey for Children and Adolescents (KiGGS)}

\section{Zusammenfassung}

Parent interviews with regard to their children's accidents and to accident protective measures in the Health Interview and Examination Survey for Children and Adolescents (KiGGS) aimed at extending our knowledge of age- and gender-specific injuries and to identify risk groups and risk factors for injury prevention. The parents of 16,706 children (aged 1 17 years) were asked about their children's injuries within the last 12 months which were medically treated, and about accident mechanisms, consequences of injuries, and ambulatory and hospital treatment. In addition, parents and children aged 11 to 17 years $(n=6813)$ were asked to give information on protective measures. According to the parents $15.9 \%$ of the children had at least one injury within the last 12 months, $15.2 \%$ because of an accident and $0.8 \%$ because of assault. In the age group 1-17 boys have been injured significantly more often than girls (17.9\% vs. $14,0 \%$ ). Overall, $13.3 \%$ of 2,410 injured children and adolescents were hospitalized. Two thirds of the accidents among toddlers were domestic accidents (60\%) whereas leisure and sport accidents were most prevalent in children and adolescents aged 5-14 years and $15-17$ years ( $32.1 \%$ and $38.9 \%$ ). The proportion of accidents in child care facilities and educational institutions tripled from infancy to school age (age 5-14 years) (10.9-28.7\%), as did traffic accidents (5.6-16.7\%). The three most frequent injury mechanisms in the age range 1-17 years were falls on level ground (35.2\%), falls from heights (25.2\%) and collisions with objects or persons (20.6\%). Falls from heights showed the highest risk in toddlers (35.8\%). Contusions, sprains and strains increased to a highest level of $50.9 \%$ in adolescents; likewise, bone fractures increased from $10.7 \%$ in toddlers to $21.8 \%$ in adolescents aged $15-17$ years. An influence of socioeconomic status on injuries overall and on consequences of injuries was not seen. For traffic accidents in children aged $1-17$ years boys $(p=0.019)$ and girls $(p=0.047)$ from families with lower socioeconomic status showed higher rates of accidents than children from families with higher socioeconomic status. The application of protective measures was lowest in the age group 14-17 years. While according to the parents about $90 \%$ of children aged 3-4 years wear a helmet when riding a bicycle or when skating, this quote was lower in the age group 5-14 $(60 \%)$ and dropped to about $15 \%$ in the age group 14-17 (about 15\%). Also the rate for using protective clothes was lowest in age group 14-17 (boys 41.8\%; girls 52.2\%). In children and adolescents the rate of selfreported helmet use is lower than estimated by their parents. In all age groups migration background and low socioeconomic status were associated with lower use of protective measures (helmets and protective clothes). The age related data analysis should be the starting point in prevention measures for specific risk groups considering migration and socioeconomic status. Prevention activities in traffic should focus on families with low social status. Adolescents should be specifically and adequately addressed regarding the benefits of certain safety measures when riding a bicycle and when skating.

\section{Schlüsselwörter}

Health Survey · Children · Adolescents . Injuries - Falls - Socioeconomic status . Migration status $\cdot$ Helmet rates · Protective measures
Hof). Während die Unfälle zu Hause mit zunehmendem Alter der Kinder abnahmen, stiegen die Spiel-, Sport- und Freizeitunfälle von 19,0 \% bei den Ein- bis unter 5-Jährigen auf das 2-Fache $(38,9 \%)$ bei den 15- bis unter 18-jährigen Jugendlichen an. Die Betreuungseinrichtungen sind bei den Ein- bis unter 5-Jährigen der dritthäufigste Unfallort. Das trifft ebenso für die Bildungseinrichtungen zu, und zwar für die Altersgruppe der 5- bis unter 15-Jährigen, jedoch mit einem deutlich höheren Anteil von $28,3 \%$ (• Abb. 2). Unfälle auf öffentlichen Verkehrswegen nahmen in allen Altersgruppen den vierten Platz ein. Ihr Anteil erhöhte sich jedoch um das 3-Fache vom Kleinkindalter (5,6\%) zum Jugendalter (16,7\%).

\section{Unfallmechanismen}

Sturzunfälle waren bei den Ein- bis unter 18-Jährigen mit einem Anteil von 60,4\% an den Unfällen insgesamt der am häufigsten genannte Unfallmechanismus. Auf Platz 2 folgte mit 20,6\% der Zusammenstoß bzw. der Zusammenprall mit Gegenständen oder Personen, gefolgt auf Platz 3 von Unfällen im Straßenverkehr $(8,3 \%)$ (• Abb. 3).

Der Anteil der Stürze aus der Höhe betrug im Kleinkindalter $35,8 \%$ und reduzierte sich bei den 15- bis unter 18-Jährigen auf $18,8 \%$. Im Kleinkindalter (ein bis 4 Jahre) waren unter den Sturzunfällen am häufigsten Stürze von der Treppe (10,4\%) und Stürze von Spielgeräten ( $7,7 \%)$. In den höheren Altersgruppen nahmen die Stürze von Treppen ab und die Stürze von Spiel- und Sportgeräten zu. Unfälle durch Zusammenstoß bzw. Zusammenprall stiegen vom Kleinkindalter zum Jugendalter stark an (ein bis 4 Jahre: $16,4 \%$; 15-17 Jahre: $24,6 \%$ ). Dabei waren Zusammenstöße mit Gegenständen im Kleinkindalter mit 13,2 \% am häufigsten. Zusammenstöße bzw. Zusammenprall mit Personen zeigen eine starke Zunahme zum Jugendalter (5-14 Jahre: 7,1\%; 15-17 Jahre: 12,7\%).

\section{Verletzungsfolgen}

Die häufigsten 4 Verletzungsfolgen bei Kindern und Jugendlichen (1-17 Jahre) waren Prellungen, Verrenkungen und Zerrungen $(33,0 \%)$, offene Wunden 
Abb. 2 Prozentualer Anteil der 4 häufigsten Unfallorte an den Unfallorten insgesamt $\left(\mathrm{N}_{\text {gewichtet }}=\mathbf{2 4 1 0}\right.$ verletzte Kinder und Jugendliche im Alter von einem bis unter 18 Jahren) $\square$ zu Hause $\square$ Sport/Freizeit/sonst. $\square$ Betreuungs-/Bildungseinrichtungen. $\square$ öffentl. Verkehrswege $\square$ o. Angabe

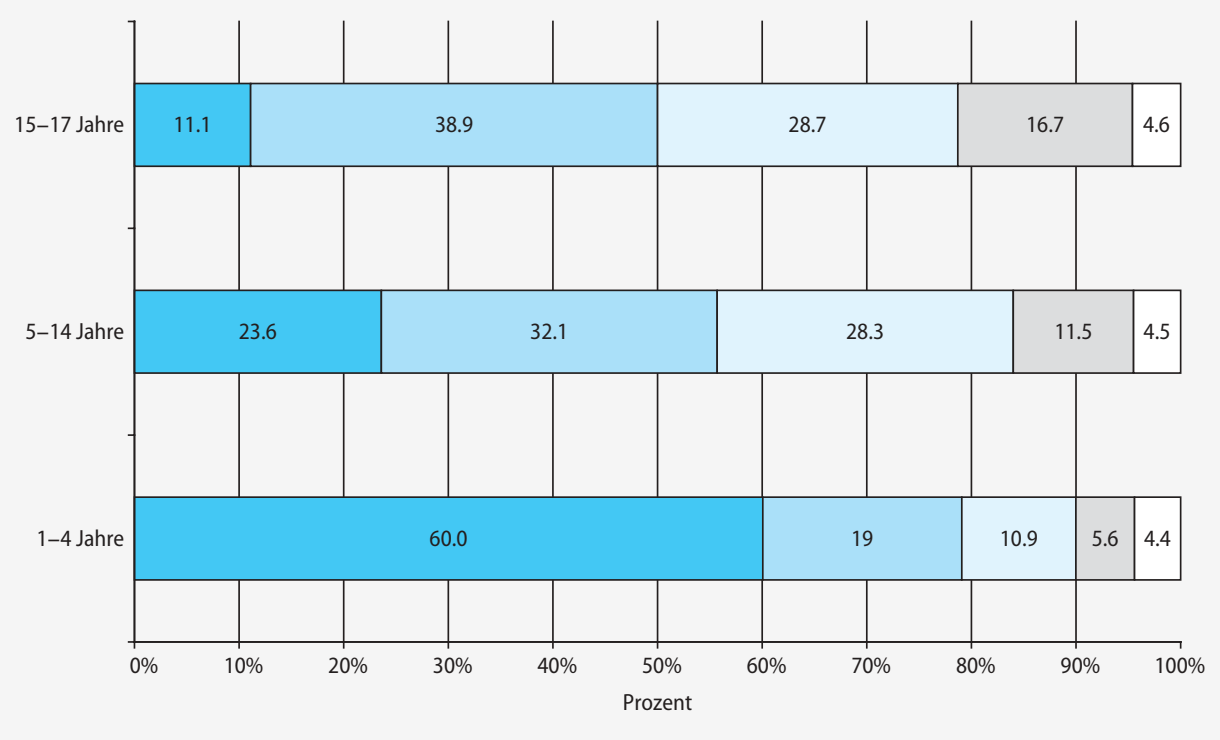

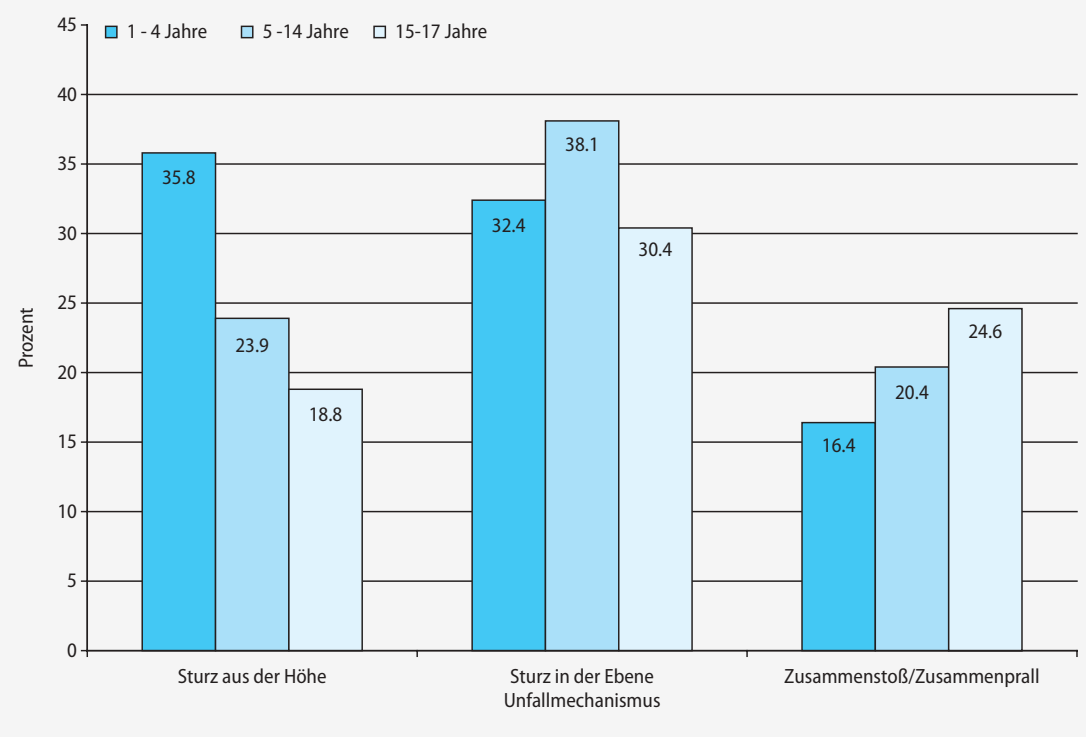

Abb. $3 \Delta$ Prozentualer Anteil der 3 häufigsten Unfallmechanismen an den Verletzungen insgesamt nach Alter ( $\mathbf{N}_{\text {gewichtet }}=\mathbf{2 4 1 0}$ verletzte Kinder und Jugendliche im Alter von einem bis unter 18 Jahren)

$(28,1 \%)$, Knochenbrüche $(19,8 \%)$ und Gehirnerschütterungen (5,7\%) (• Abb.4). Die Analyse der Verletzungsfolgen nach Altersgruppen zeigte eine Altersdynamik: Prellungen, Verrenkungen und Zerrungen erhöhten sich stark über alle Altersgruppen hinweg mit einem Häufigkeitsgipfel von 50,9\% in der Gruppe aller verletzten Jugendlichen, während offene Wunden vom Kleinkindalter zum Jugendalter um die Hälfte abnahmen (von
$37,7 \%$ auf $17,9 \%)$. Ebenso stieg der Anteil an Knochenbrüchen von 10,7\% bei verletzten Kleinkindern auf ca. 22,8 \% bei den 5- bis unter 18-Jährigen. Geschlechtsspezifische Unterschiede ließen sich für offene Wunden $(\mathrm{p}<\mathrm{0}, 001)$, Knochenbrüche $(\mathrm{p}=0,024)$ und Gehirnerschütterungen $(\mathrm{p}=\mathrm{o}, 045)$ nachweisen, d. h., Jungen waren stärker betroffen als Mädchen, jedoch nicht bei Prellungen, Verrenkungen und Zerrungen.
Soziale Lage, Migrationsstatus und Verletzungen

Eine Analyse der 3 häufigsten Unfallmechanismen nach Sozialstatus zeigte nur bei den Verkehrsunfällen, dass Kinder und Jugendliche mit niedrigem Sozialstatus signifikant häufiger Verkehrsunfälle hatten als Kinder und Jugendliche mit einem hohen Sozialstatus. Dies traf auch signifikant für Mädchen ( $\mathrm{p}=0,058)$ und Jungen $(p=0,018) z u(\bullet A b b .5)$. Die Analyse der Verkehrsunfälle nach Sozialstatus für Kinder mit Migrationshintergrund ergab aufgrund der geringen Anzahl der durch Verkehrsunfälle verletzten Kinder keine verwertbaren Aussagen.

Bei den Unfällen insgesamt konnte nur bei den 5- bis unter 15-jährigen Migrantenkindern eine signifikant niedrigere Verletzungshäufigkeit festgestellt werden $(\mathrm{p}<0,005)$ als bei den deutschen Schülern. Wurde nach dem Geschlecht differenziert, zeigte sich, dass die niedrigere Verletzungshäufigkeit bei Kindern 5 bis unter 15 Jahren nur bei den Mädchen mit Migrationshintergrund signifikant war $(\mathrm{p}<\mathrm{0,001})$. Anders verhielt es sich in der Altersgruppe der Kleinkinder. Hier bestanden signifikant höhere Verletzungsraten $(p=0,032)$ für die Jungen mit Migrationshintergrund verglichen $\mathrm{zu}$ ihren deutschen Altersgefährten (• Abb. 6). 


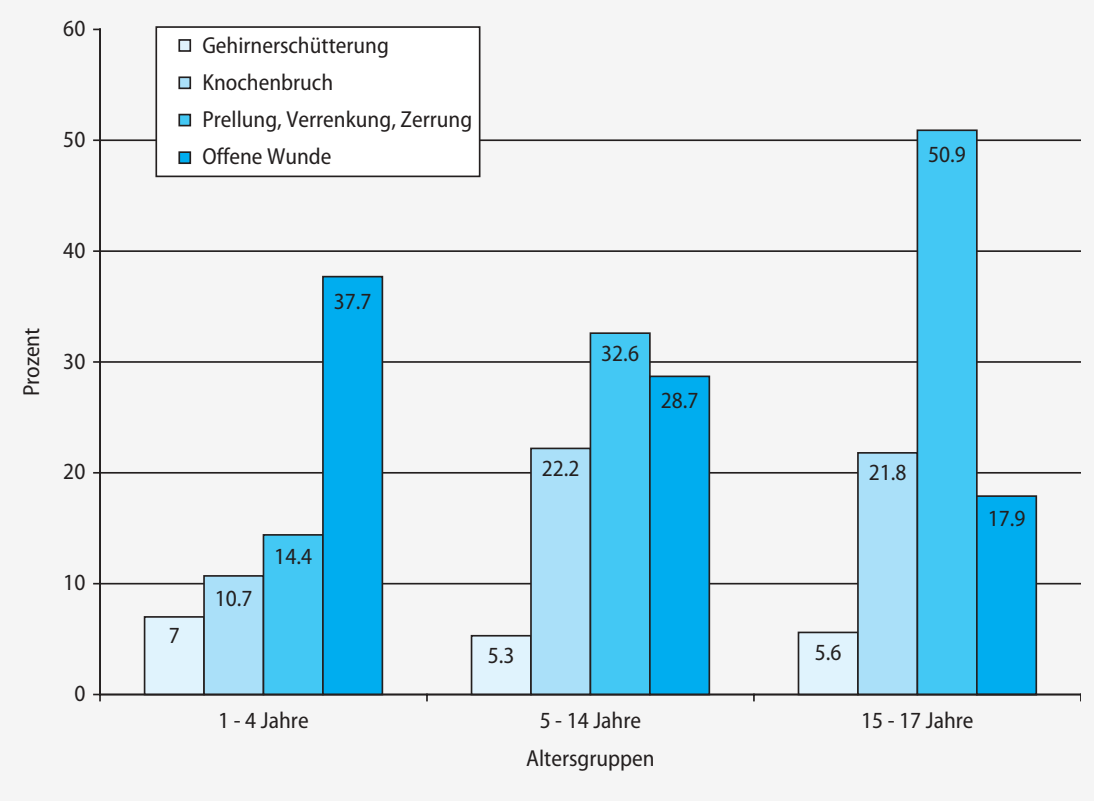

Abb. $4 \Delta$ Prozentualer Anteil der $\mathbf{4}$ häufigsten Verletzungsfolgen nach Alter $\left(\mathbf{N}_{\text {gewichtet }}=\mathbf{2 4 1 0}\right.$ verletzte Kinder und Jugendliche im Alter von einem bis unter 18 Jahren)

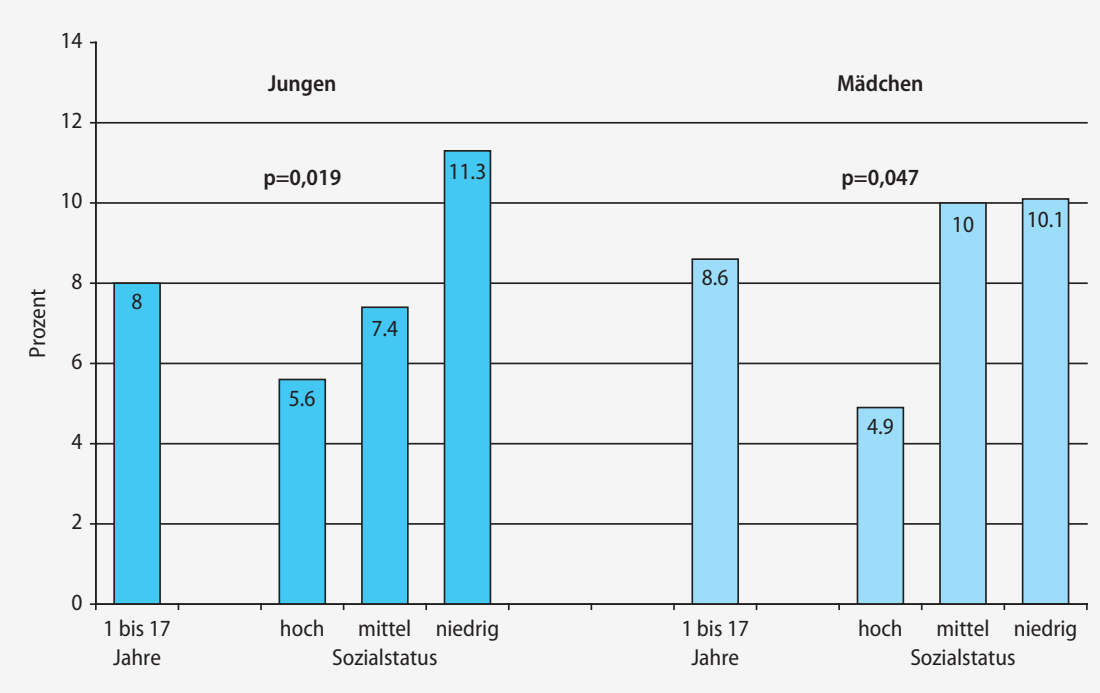

Abb. 5 P Prozentualer Anteil der Verkehrsunfälle nach Geschlecht und Sozialstatus ( $\mathrm{N}_{\text {gewichtet }}=\mathbf{2 4 1 0}$ verletzte Kinder und Jugendliche im Alter von einem bis unter 18 Jahren)

\section{Schutzmaßnahmen durch Helm und Protektoren}

Nach den Angaben der Eltern fuhren über $90 \%$ der Kinder Fahrrad (3-4 Jahre: 82,1\%; 5-14 Jahre: 97,2 \%; 15-17 Jahre: 89,8\%). Mit Inlineskates waren $67,5 \%$ der 5 - bis unter 15-Jährigen und 51,7\% der 15 - bis unter 18-Jährigen in ihrer Freizeit unterwegs. Die persönlichen Schutzmaßnahmen wurden in den verschiedenen Altersgruppen sehr unterschiedlich umgesetzt. beim Fahrradfahren gab es für alle Altersgruppen ab 3 Jahren signifikante Unterschiede mit niedrigeren Quoten bezogen auf die Region Ost (inklusive Berlin) $(p<0,001)$, einem niedrigen Sozialstatus $(\mathrm{p}<0,001)$ und dem Migrationsstatus $(\mathrm{p}<\mathrm{o,001})(\bullet$ Tabelle 1$)$.

\section{Diskussion}

Mit dem KiGGS liegen erstmals für ein- bis unter 18-jährige Kinder und Jugendliche bundesweite repräsentative Daten über Verletzungen durch Unfälle und Gewalt vor, die ärztlich behandelt werden mussten. Die durchschnittliche Verletzungsrate von $15,9 \%$ weist auf eine hohe Krankheitslast für das Kindes- und Jugendalter hin. Vergleichbare bevölkerungsbezogene Angaben zu Verletzungen liegen in Deutschland nur aus dem gemeindebezogenen Monitoring in der Stadt Delmenhorst vor [7]. Dabei müssen bei diesem Vergleich die unterschiedlichen Erhebungsmethoden berücksichtigt werden, d. h. einerseits durch Befragung der Eltern retrospektiv zu Unfällen der Kinder in den vergangenen 12 Monaten wie im KiGGS, andererseits durch direkte Erfassung im Zusammenhang mit der ärztlichen Krankenhausbehandlung (Notfallambulanz und stationär) wie in Delmenhorst. In Delmenhorst lagen die Verletzungsraten mit 18,6\% bei ein- bis unter 5-jährigen Kleinkindern am höchsten und bei Schülern (5-14 Jahre) mit 12,5\% deutlich niedriger. Die KiGGS-Ergebnisse zeigten dagegen eine genau umgekehrte Altersdynamik. Möglicherweise lassen sich diese Differenzen über eine unterschiedliche Inanspruchnahme des stationären und ambulanten Versorgungssystems erklären, d. h., Eltern von verletzten kleinen Kindern suchen bei Verletzungen eher die Notfallambulanz des Krankenhauses auf als Jugendliche bei Verletzungen.

Verletzungen durch Gewalt hatten den höchsten Anteil bei Schülern (5-14 Jahre), sowohl für Mädchen als auch für Jungen. Die KiGGS-Ergebnisse ermittelten eine populationsbezogene Rate von $1,1 \%$ bei Jungen und o,8\% bei Mädchen. Eine Analyse zu Raufunfällen an Schulen durch den Bundesverband der Unfallkassen kam zu vergleichbaren Raten von insgesamt 11,3 Raufunfällen auf 1000 Schüler. 


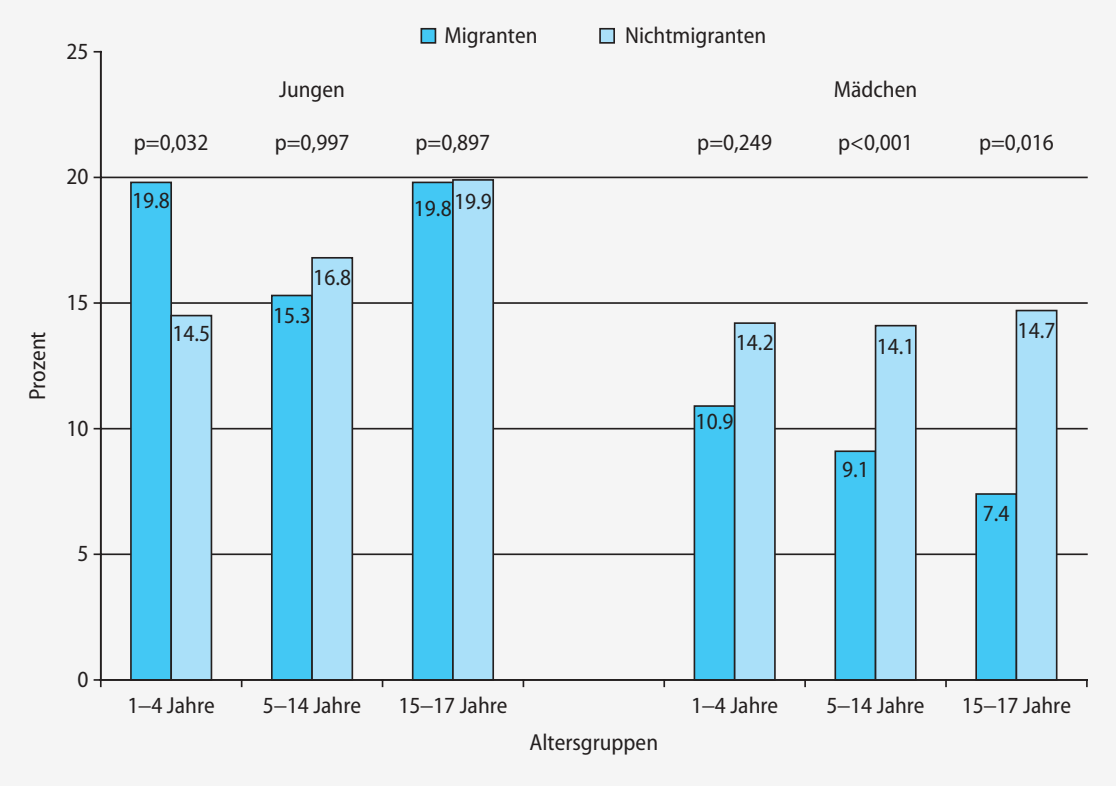

Abb. $6 \Delta$ Verletzungen durch Unfälle nach Alter, Geschlecht und Migrationsstatus ( $\mathrm{N}_{\text {gewichtet }}=\mathbf{2 4 1 0}$ verletzte Kinder und Jugendliche im Alter von einem bis unter 18 Jahren)

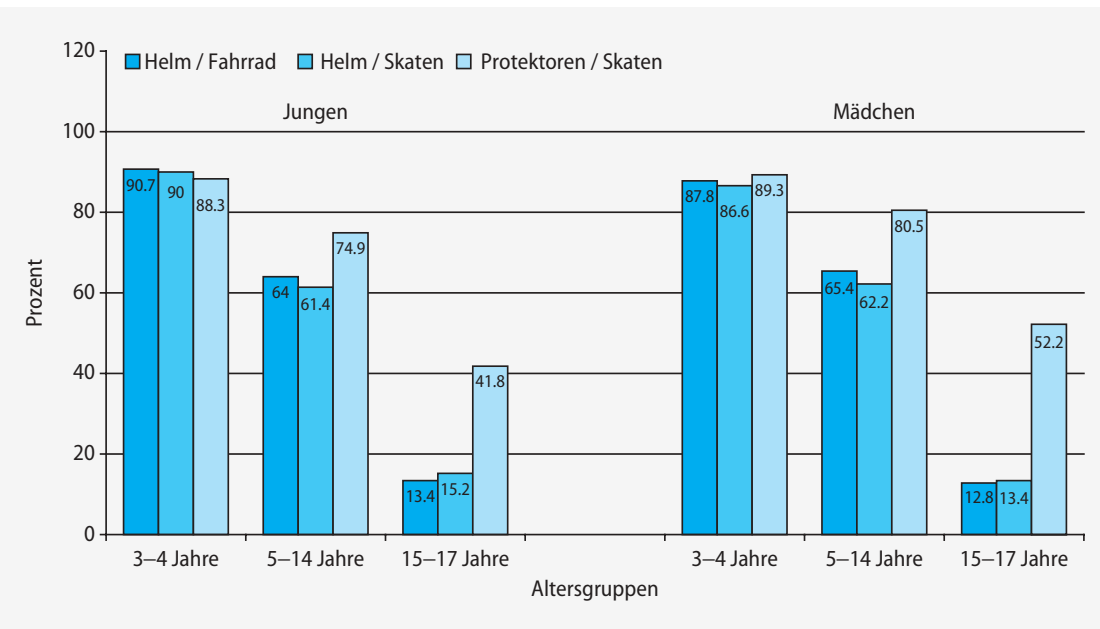

Abb. $7 \Delta$ Helmtragequoten beim Fahrradfahren (Elternangaben: 13.452) und Inlineskaten (Elternangaben: 7692) sowie Schutzmaßnahmen durch Protektoren (Elternangaben: 8259) nach Alter und Geschlecht

Dabei zeigte der Häufigkeitsgipfel bei den Hauptschulen eine 3-fach höhere Quote [31].

Der Anteil der stationär behandelten Kinder an allen verletzten Kindern betrug in Delmenhorst für Kinder unter 15 Jahren 6,4\%, im KiGGS war dieser Anteil mehr als doppelt so hoch (13,3\%) für die Ein- bis unter 18-Jährigen. Die Bundesanstalt für Arbeitsschutz und Arbeitsmedizin (BAuA) ermittelte in ihrem zuletzt im Jahr 2000 durchgeführten Survey von Heim- und Freizeitunfällen sogar einen Anteil von 15\% stationären und $85 \%$ ambulant behandelten Kindern [32]. Die höheren Anteile von stationär behandelten Kindern und Jugendlichen in den jeweiligen Surveys könnten beispielsweise darauf zurückzuführen sein, dass sich Eltern eher an die Krankenhausbehandlungen ihrer Kinder erinnern als an weniger schwere ambulant ärztlich behandelte Verletzungen. Andererseits spiegeln auf das Krankenhaus bezogene Daten immer auch die Versorgungslandschaft wider. So ist die Kinderklinik in Delmenhorst im Auftrag der KV Anlaufstelle für zu behandelnde
Kinder, wenn Kinderarztpraxen keine Sprechstunden mehr haben.

Die in Deutschland publizierten epidemiologischen Analysen zu Kinderunfällen auf der Basis der Todesursachenstatistik $[1,20]$, der kinderärztlichen Schuleingangsuntersuchungen $[8,9]$ und des Unfallmonitorings in Delmenhorst zeigten übereinstimmend [6, 7], dass der häufigste Unfallort bei den ein- bis unter 5-jährigen Kindern der häusliche Bereich war. Mit zunehmendem Alter und Aktionsradius der Kinder verlagerte sich dann der Unfallort in Richtung Bildungseinrichtungen und in den Freizeit-, Sport- sowie Verkehrsbereich. Dieses altersspezifische Unfallgeschehen fand sich auch bei den Ergebnisse der BAuA über Heim- und Freizeitunfälle [32, 33]. Die KiGGS-Daten bestätigen diese Ergebnisse und zeigen darüber hinaus eine weitere Zunahme der Freizeit- und Sportunfälle für das Jugendalter (15-17 Jahre).

Bei den Verletzungsmechanismen nahmen im KiGGS die Sturzunfälle bei allen Altersgruppen den ersten Rang ein. Während bei Kleinkindern (ein bis 4 Jahre) die Stürze aus der Höhe, darunter insbesondere Stürze von Treppen, am häufigsten waren, nahmen mit zunehmendem Alter die Stürze von der Höhe ab und die auf der Ebene zu. Im Jugendalter standen bei den Stürzen aus der Höhe Spiel- und Sportgeräte im Vordergrund. Zu vergleichbaren Ergebnissen kam eine epidemiologische Studie zu Sturzunfällen im Kindesalter, die die Daten aus Betreuungs- und Bildungseinrichtungen der Bundesunfallkasse mit einschloss [34]. Weitere differenziertere produktbezogene Aussagen bei den Sturzunfällen lassen sich über die KiGGS-Daten nicht machen, da diese nicht erfragt wurden. Diese Lücke sollte in Zukunft auch in Deutschland über Daten aus der Injury Data Base (IDB) geschlossen werden. Das heißt, auch in Deutschland sollte wie in anderen europäischen Ländern ein krankenhausbezogenes Monitoring von Verletzungen etabliert werden, das die unfallverursachenden Produkte mit erfasst [35].

Eine umfangreiche epidemiologische Analyse der Kinderunfälle nach Geschlecht auf der Basis verschiedenster in Deutschland verfügbarer Datenquellen zu Mortalität und Morbidität konnte zeigen, 
Tabelle 1

Helmtragequoten beim Fahrradfahren und Inlineskaten und Tragequoten von Protektoren (Arm- und Knieschonern) beim Inlineskaten nach Geschlecht, Region, Sozialstatus und Migrationsstatus

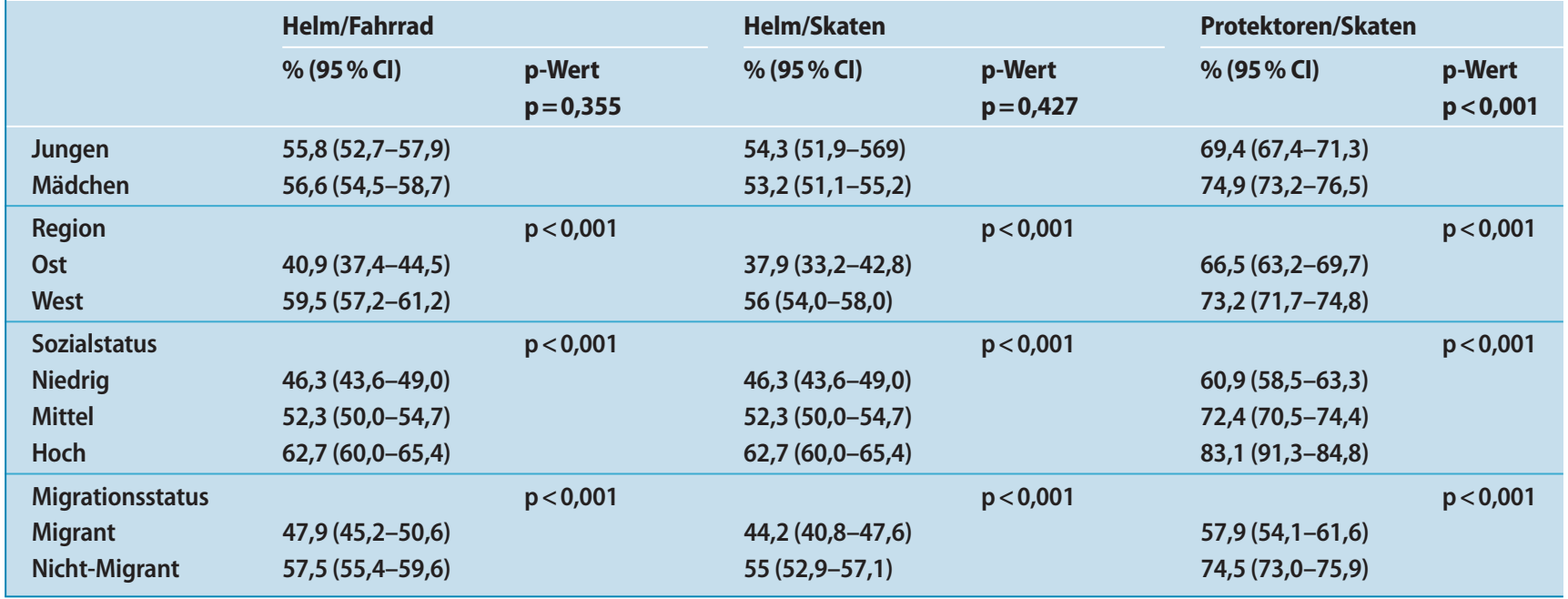

dass für jedes Alter $>1$ Jahr galt, dass Jungen sich häufiger als Mädchen verletzten [6]. Dieser starke Geschlechtsunterschied konnte ebenso ausgeprägt bei den KiGGSDaten gefunden werden. Obwohl die Ursachen für die geschlechtsspezifischen Unfallmechanismen nach der internationalen Literatur auch unklar sind, wurden Hypothesen aufgestellt [17]. Einerseits werden die unterschiedlichen angeborenen motorischen Fähigkeiten diskutiert, andererseits das Verhalten, das wiederum von den Sozialisationsbedingungen abhängt, d. h. von der unterschiedlichen Risikobereitschaft und der unterschiedlichen Exposition gegenüber Gefahren. Dies wird von der Beobachtung gestützt, dass der Unterschied zwischen Unfällen bei Jungen und Mädchen am größten für Aktivitäten ist, die von den Kindern selbst ausgehen, wie Fahrradfahren, Schwimmen, auf der Straße spielen, während der Geschlechtsunterschied kaum noch besteht, wenn das Kind keinen Einfluss auf die Exposition gegenüber Gefahren hat, wie z. B. als Beifahrer im Pkw.

In Deutschland werden in den amtlichen Statistiken, wie z. B. der Krankenhausdiagnose-, Straßenverkehrs- und Schülerunfallstatistik, keine Sozialdaten und auch nicht der Migrationsstatus erhoben. Somit können keine Aussagen zu sozioethnischen Risiken gemacht werden. Die WHO wies in ihrem 2005 publizierten Bericht zu „Verletzungen und Gewalt in Europa" explizit darauf hin, dass Migranten, Flüchtlinge und Kinder aus $\mathrm{Fa}$ milien mit niedrigem soziökonomischem Status ein besonderes Verletzungsrisiko hatten [2]. Eine viel zitierte englische Studie konnte zeigen, dass Kinder von Eltern mit dem niedrigsten beruflichen Status ein 16-mal größeres Risiko hatten, an einem Wohnungsbrand, und ein 5-mal größeres Risiko als Fußgänger zu sterben, als Kinder von Eltern mit dem höchsten beruflichen Status [18]. In Deutschland konnten diese Zusammenhänge zwischen Sozialstatus und bestimmten Verletzungsmechanismen erst dargestellt werden, als in bevölkerungsbezogenen Erfassungssystemen auch soziale Angaben mit erhoben wurden (Einschulungsuntersuchungen im Land Brandenburg seit 1994, im Land Schleswig-Holstein seit 2002 und Unfallmonitoring der Stadt Delmenhorst 1998-2002). Im KiGGS wurde sowohl der Sozial- als auch Migrantenstatus differenziert erhoben. Die Datenanalyse zu Unfällen nach Sozialstatus konnte einen signifikanten Zusammenhang zwischen höherer Unfallhäufigkeit der Kinder und niedrigem Sozialstatus nur bei den Straßenverkehrsunfällen für Mädchen und Jungen ermitteln. Analoge Ergebnisse wurden im Land Brandenburg bei den Einschulungskindern festgestellt [10].

Die KiGGS-Ergebnisse zu den Schutzmaßnahmen zeigten differenzierte Aussagen über Tragequoten von Helmen und
Protektoren ab 3 Jahren aufwärts, wie sie bisher noch nicht in Deutschland existieren. Die Bundesanstalt für Straßenwesen (BAST) erhebt seit 1992 kontinuierlich bundesweit die Helmtragequoten beim Fahrradfahren durch Beobachtungen im Straßenverkehr, jedoch liegen aktuelle Helmtragequoten für 2005 nur für unter 10-Jährige mit insgesamt $53 \%$ vor. Aussagen zu Helmtragequoten nach Sozialstatus bzw. Migrationstatus konnten von der BAST mangels Datenlage nicht gemacht werden. Neu sind daher die Erkenntnisse der KiGGS-Daten, die deutlich machen, dass das Tragen von Helmen beim Fahrradfahren und Inlineskaten von Kindern mit Migrationshintergrund und aus Familien mit niedrigem Sozialstatus signifikant weniger umgesetzt wird. Soziale Unterschiede sind auch beim Durchsetzen der Schutzmaßnahmen beim Fahrradfahren und beim Inlineskaten zu erkennen, wobei Kinder aus Familien mit niedrigem Sozialstatus, aus der Region Ost auch die Kinder mit Migrationshintergrund weniger vor Verletzungen geschützt sind als ihre sozial besser gestellten Altersgefährten.

\section{Schlussfolgerungen und Ausblick}

Die altersgruppenbezogene Datenanalyse sollte Ausgangspunkt für zielgruppenbezogene Präventionsmaßnahmen sein. Bei der Zielgruppe der Kleinkinder 
[36] muss das häusliche Unfallgeschehen im Fokus der Prävention stehen und bei Schulkindern der Freizeit- und Sportunfall. Darüber hinaus sind Präventionsmaßnahmen auf geschlechtsspezifische Besonderheiten auszurichten und der Sozial- und Migrationsstatus bei der Prävention von Unfällen im Straßenverkehrsbereich besonders zu beachten. Jugendliche sollten bei der Aufklärung über den Nutzen von Schutzmaßnahmen beim Fahrradfahren und Skaten verstärkt und adäquat angesprochen werden. Denn nur die Information an Eltern über sinnvolle Schutzmaßnahmen reicht für Prävention nicht aus. Dies zeigt die Diskrepanz zwischen den Angaben der Kinder und Jugendlichen zu den Eltern. Die weitere Auswertung der Verletzungsdaten wird mögliche Einflussfaktoren wie Koordination, psychische Parameter, soziale Faktoren, Schulklima, Schultyp vertiefend untersuchen.

\section{Korrespondierende Autorin}

\section{Dr. Gabriele Ellsäßer}

Landesgesundheitsamt Brandenburg im LASV Wünsdorfer Platz 3

15806 Zossen, BRD

E-Mail: Gabriele.Ellsaesser@lga.brandenburg.de

\section{Literatur}

1. Ellsäßer G, Berfenstam R (2000) International comparisons of child injuries and prevention programs: recommendations for an improved prevention program in Germany. Inj Prev 6:41-45

2. World Health Organization (2005) Injuries and violence in Europe. Why they matter and what can be done. WHO Regional Office for Europe, Kopenhagen

3. UNICEF (2001) A league table of child deaths by injury in rich nations. Innocenti Report Card No2, UNICEF Innocenti Research Centre, Florence

4. Mulder S, van Beeck EF (1999) Measuring the burden of injuries. European Consumer Safety Association, Consumer Safety Institute (Hrsg), Amsterdam

5. Rogmans WHJ, Mulder S (1998) Measuring the severity and costs of accidental injuries. European Consumer Safety Association, Consumer Safety Institute (Hrsg), Amsterdam

6. Ellsäßer G (2006) Epidemiologische Analyse bei Kindern unter 15 Jahren in Deutschland - Ausgangspunkt für die Unfallprävention. Gesundheitswesen 68:421-428

7. Böhmann J, Ellsäßer G (2004) Bevölkerungsbezogenes Unfallmonitoring von Kinderunfällen in einer deutschen Gemeinde. Monatsschr Kinderheilkd 152:299-306
8. Ministerium für Soziales, Gesundheit und Verbraucherschutz (2005) Bericht über die Untersuchung des Kinder- und Jugendärztlichen Dienstes in Schleswig-Holstein im Jahr 2004. Eigenverlag, Kiel

9. Ministerium für Arbeit, Soziales, Gesundheit und Familie (2007) Wir lassen kein Kind zurück. Soziale und gesundheitliche Lage von kleinen Kindern im Land Brandenburg. Beiträge zur Sozial- und Gesundheitsberichterstattung Nr. 5. Eigenverlag, Potsdam

10. Ellsäßer G (2000) Injuries among preschool children in the federal state of Brandenburg depending on social factors - 1997-99. International Conference, Social inequities in injury risks, book of abstracts, Paris

11. Ellsäßer G, Böhm A, Kuhn J et al. (2002) Soziale Ungleichheit und Gesundheit bei Kindern. Ergebnisse und Konsequenzen aus den Brandenburger Einschulungsuntersuchungen. Kinderärztl Praxis 4:248-257

12. Ellsäßer G, Böhmann J (2004) Thermische Verletzungen im Kindesalter ( $<15$ Jahre) und soziale Risiken. Kinderärztl Praxis 2:34-38

13. Ellsäßer G, Böhmann J (2002) Thermische Verletzungen im Kindesalter und soziale Risiken, Präventionsziele. In: Geene R, Gold C, Hans C (Hrsg) Armut und Gesundheit, Gesundheitsziele gegen Armut. Netzwerke für Menschen in schwierigen Lebenslagen. Teil 1, Materialien zur Gesundheitsförderung Band 10. Gesundheit Berlin e.V., Berlin

14. Petridou E, Anastasiou A, Katsiardanis K et al. (2005) A prospective population based study of childhood injuries: the Velestino town study. Eur J Public Health 15:9-14

15. Christie N (1995) The high risk child pedestrian, socio-economic and environmental factors in their Laboratory accidents. Transport Research Laboratory. Project 117, Crowthorne

16. Dowswell T, Towner E (2002) Social deprivation and the prevention of unintentional injury in childhood: a systematic review. Health Education Res 17:221-237

17. Laflamme L (1998) Social inequality in injury risks. Sweden's Knowledge accumulated and plans for the future. National Institute of Public Health 33

18. Roberts I, Power C (1996) Does the decline in child injury death rates vary by social class? BMJ 313 : 784-786

19. Ellsäßer G, Diepgen TL (2002) Epidemiologische Analyse von Sturzunfällen im Kindesalter (<15 Jahre). Konsequenzen für die Prävention. Bundesgesundheitsbl Gesundheitsforsch Gesundheitsschutz 45:267-276

20. Ellsäßer G (1998) Daten für Taten. Fakten zur Prävention von Kinderunfällen. Forum Unfallprävention im Deutschen Grünen Kreuz (Hrsg) 1. Aufl. Verlag im Kilian, Marburg

21. Bundesanstalt für Straßenwesen (2003) Bericht: Sicherung durch Gurte, Helme und andere Schutzsysteme 2003. Kontinuierliche Erhebungen zum Schutzverhalten von Verkehrsteilnehmern. Bericht zu FP 82.251/2003, http://www.bast.de/htdocs/ veroeffentlichung/bastinfo/info2004/info0504. htm

22. Bundesanstalt für Straßenwesen (2005) Bericht: Sicherung durch Gurte, Helme und andere Schutzsysteme 2005. Kontinuierliche Erhebungen zum Schutzverhalten von Verkehrsteilnehmern. Bericht zu FP 82.279/2004, http://www.bast.de/htdocs/ veroeffentlichung/bastinfo/info2006/info0504. htm
23. Holder Y, Peden M, Krug E et al. (2001) Injury Surveillance Guidelines. World Health Organisation, Geneva, http://www.who.int/violence_ injury_prevention/index.html

24. Kurth B-M (2007) Der Kinder- und Jugendgesundheitssurvey (KiGGS): Ein Überblick über Planung, Durchführung und Ergebnisse unter Berücksichtigung von Aspekten eines Qualitätsmanagements. Bundesgesundheitsbl Gesundheitsforsch Gesundheitsschutz 50:533-546

25. Kamtsiuris $P$, Lange $M$, Schaffrath Rosario A (2007) Der Kinder- und Jugendgesundheitssurvey (KiGGS): Stichprobendesign, Response und Nonresponse-Analyse. Bundesgesundheitsbl Gesundheitsforsch Gesundheitsschutz 50:547-556

26. Hölling H, Kamtsiuris P, Lange M et al. (2007) Der Kinder- und Jugendgesundheitssurvey (KiGGS): Studienmanagement und Durchführung der Feldarbeit. Bundesgesundheitsbl Gesundheitsforsch Gesundheitsschutz 50:557-566

27. Filipiak-Pittroff B, Wölke G (2007) Externe Qualitätssicherung im Kinder- und Jugendgesundheitssurvey (KiGGS). Vorgehensweise und Ergebnisse. Bundesgesundheitsbl Gesundheitsforsch Gesundheitsschutz 50:573-577

28. Dölle R, Schaffrath Rosario A, Stolzenberg H (2007) Der Kinder- und Jugendgesundheitssurvey (KiGGS): Datenmanagement. Bundesgesundheitsbl Gesundheitsforsch Gesundheitsschutz 50: 567-572

29. Lange $M$, Kamtsiuris $P$, Lange $C$ et al. (2007) Messung soziodemographischer Merkmale im Kinderund Jugendgesundheitssurvey (KiGGS) und ihre Bedeutung am Beispiel der Einschätzung des allgemeinen Gesundheitszustands. Bundesgesundheitsbl Gesundheitsforsch Gesundheitsschutz 50:578-589

30. Schenk L, Ellert U, Neuhauser H (2007) Kinder und Jugendliche mit Migrationshintergrund in Deutschland. Methodische Aspekte im Kinderund Jugendgesundheitssurvey (KiGGS). Bundesgesundheitsbl Gesundheitsforsch Gesundheitsschutz 50:590-599

31. Bundesverband der Unfallkassen (2003) Gewalt an Schulen. Ein empirischer Beitrag zum Gewalt verursachten Verletzungsgeschehen an Schulen in Deutschland 1993-2003. Eigenverlag, München

32. Kreileder M, Holeczek M (2002) Unfallverletzungen in Heim und Freizeit im Jahr 2000. Sonderschrift S74 der Baut, Dortmund Berlin Dresden

33. Henter A (2001) Unfälle in Heim und Freizeit im Kindes- und Jugendalter 1996. Sonderauswertung. Bundesanstalt für Arbeitsschutz und Arbeitsmedizin, Dortmund

34. Ellsäßer G, Diepgen TL (2002) Epidemiologische Analyse von Sturzunfällen im Kindesalter ( $<15$ Jahre). Konsequenzen für die Prävention. Bundesgesundheitsbl Gesundheitsforsch Gesundheitsschutz 45:267-276

35. Austrian Road Safety Board (2006) Injuries in the European Union. Statistics summary 2002-2004. Wien

36. Ellsäßer G (2006) Wie können Pädiater Eltern für die Unfallprävention motivieren. Kinderärztl Praxis 77:344-352 